\title{
Up-regulation of DGAT1 in cancer tissues and tumor-infiltrating macrophages influenced survival of patients with gastric cancer
}

Ping $\mathrm{He}^{1+}$, Shihuan Cheng ${ }^{2+}$, Feng $\mathrm{Hu}^{3}$, Zhanchuan $\mathrm{Ma}^{4,5^{*}}$ (D) and Yan $\mathrm{Xia}^{1 *}$

\begin{abstract}
Background: Diacylglycerol-acyltransferase 1 (DGAT1) plays an important role in the energy storage and is involved in cancer progression. A growing number of evidences showed that elevated expression of DGAT1 in cancer tissue indicated a poor outcome in cancer patients. However, the relationship between DGAT1 and gastric cancer is still unclear. Thus, Transcriptomic analysis and in vitro experiments were performed to investigate the role of DGAT1 in gastric cancer, as well as the potential therapy target in gastric cancer treatment.

Methods: We screened the public cancer datasets to identify the expression and function of DGAT1 in gastric cancer and tumor infiltrating lymphocytes. Then we testified the DGAT1 expression and function after sodium oleate treatment in AGS and MKN45 cell line. Finally, we analyzed ration of apoptosis, necrosis in gastric cancer cells by using flow cytometry after administration of DGAT1 inhibitor.

Results: Our results showed a highly expression of DGAT1 in gastric cancer tissues ( $n=5, p=0.0004)$, and tumorinfiltrating macrophages with elevated DGAT1 expression is associated with poor overall survival in gastric cancer patients. In addition, gastric cell lines AGS $(n=3, p<0.05)$ and MKN45 $(n=3, p<0.01)$ expressed higher level of DGAT1 than human gastric mucosal epithelial cell line GES-1. Administration of DGAT1 inhibitor effectively suppressed functional factors expression and induced cell death in MKN45.

Conclusion: The findings of this research provide an in-depth insight into the potential role and influences involved in DGAT1 in the gastric cancer patients. And higher expression of DGAT1 leads to lower overall survival (OS) rate in patients with poorly differentiated gastric cancer. Our findings suggest a potential role for DGAT1 in the gastric cancer progression and inhibiting DGAT1 might be a promising strategy in gastric cancer treatment.
\end{abstract}

Keywords: Diacylglycerol-acyltransferase 1, Gastric cancer, Prognosis of patients, Cancer treatment

\footnotetext{
*Correspondence: mazc15@mails.ju.edu.cn; 37228481@qq.com

${ }^{\dagger}$ Ping He and Shihuan Cheng contributed equally to this work.

${ }^{4}$ Central Laboratory, The First Hospital of Jilin University, Changchun, Jilin,

China

'Department of Gastroenterology, The First Hospital of Jilin University,

Changchun 130021, Jilin, China

Full list of author information is available at the end of the article
}

(c) The Author(s). 2021 Open Access This article is licensed under a Creative Commons Attribution 4.0 International License, which permits use, sharing, adaptation, distribution and reproduction in any medium or format, as long as you give appropriate credit to the original author(s) and the source, provide a link to the Creative Commons licence, and indicate if changes were made. The images or other third party material in this article are included in the article's Creative Commons licence, unless indicated otherwise in a credit line to the material. If material is not included in the article's Creative Commons licence and your intended use is not permitted by statutory regulation or exceeds the permitted use, you will need to obtain permission directly from the copyright holder. To view a copy of this licence, visit http://creativecommons.org/licenses/by/4.0/ The Creative Commons Public Domain Dedication waiver (http://creativecommons.org/publicdomain/zero/1.0/) applies to the data made available in this article, unless otherwise stated in a credit line to the data. 


\section{Background}

Cancer is a leading cause of death to human beings [1]. Gastric cancer is responsible for more than 900,000 deaths in the past year, rapidly growing number of new cases and deaths make it the third leading cause of cancer death in the world [1,2]. Also, data from The World Health Organization (WHO) demonstrated that a growing number of new cases of gastric cancer occur in developing countries [3]. Regionally, people in East Asia, East Europe, and South America have higher gastric cancer incidence [4]. And it remains a tough work to cure gastric cancer in worldwide, primarily because most patients present with advanced disease [5]. Clinical data showed that advanced stage of gastric patients have poor five-year survival rate [5]. Evidence suggests that early diagnosis is critical for gastric cancer treatment and improving sufferers' survival [6]. However, despite its extensive clinical studies, early diagnosis of gastric cancer still cannot meet the requirement due to the lack of appropriate biomarkers [6].

Generally, adipose tissue is the primary site to syntheses fatty acid in animals [7]. For example, it was reported that brown adipose tissues produced angiopoietin-like 4 play an important role in controlling lipoprotein metabolism, deficiency of which leaded to improvement of triglyceride clearance without loss of body weight in mice [8]. However, evidences showed that tumor tissue can also produce abundant fatty acid to facilitate lung tumorigenesis by upregulating long-chain family member 3 level and augmenting cellular ATP in KRAS mutant mouse model [9]. Further, an in vitro study demonstrated that accumulation of lipid droplets in colon cancer induced mitochondrial respiration indirectly promotes tumor growth and metastasis by expanding tumor-associated macrophages [10]. These studies suggest that fatty acid and the related metabolic signaling pathway might have the potential to determine the local milieu and hence tumor growth.

Triacylglycerol (TAG) is the major energy source stored in human adipose tissue [8]. Diacylglycerolacyltransferase $1(D G A T 1)$ is responsible for synthesis of TAG after utilizing two substrates diacylglycerol and fatty-acyl CoA with respect to lipid metabolism [11]. $D G A T 1$ is a transmembrane protein that can be found in the endoplasmic reticulum of several types of cells. Data from several studies suggest that suppressed $D G A T 1$ is a promising strategy to inhibit prostate cancer cell growth and, therefore, targeting DGAT1 might be conducive for clinical gastric cancer treatment [12, 13]. It has been previously reported that the elevated level of reactive oxygen species is facilitated to cancer progression and metastasis, and gastric cancer cell proliferation and migration could be suppressed after administration of inhibitor to block the reactive oxygen species production [14-16]. Another study demonstrated that accumulation of the nicotinamide adenine dinucleotide phosphate oxidase 2 (NOX2) activates Akt, Stat3, and $\mathrm{I}_{\kappa} \mathrm{B} \alpha$ signaling pathways in two gastric cancer cells. Restrained NOX2 expression decreases cyclin D1 expression and leads to cell growth arrest [16]. However, much uncertainty still exists about the relationship between DGAT1 and the reactive oxygen species in gastric cancer progression. The objectives of this research are to determine whether DGAT1 functionally influence gastric cancer progression and prognosis of patients, and uncover the clinic meaning of DGAT1.

\section{Methods \\ ONCOMINE database}

ONCOMINE database (www.oncomine.org) is a convenient online cancer database that unifies highthroughput cancer profiling data across a large volume of cancer types, subtypes, and experiments [17]. In our study, transcriptional expressions of DGAT1 between different types of cancer tissues and their corresponding adjacent normal control samples were obtained from ONCOMINE database. Difference of transcriptional expression was compared by students' t-test. Cut-off of $p$ value and fold change were as following: $p$ value: $1 \mathrm{E}-4$, fold change: 2 , gene rank: $10 \%$, data type: mRNA. The used datasets were listed as follow: Superficial Bladder Cancer, Sanchez-Carbayo Bladder 2; Myeloma, Zhan Myeloma 3; Pancreatic Adenocarcinoma, IacobuzioDonahue Pancreas 2; Ovarian Serous Cystadenocarcinoma, TCGA Ovarian; Papillary Renal Cell Carcinoma, Yusenko Renal; Gastric cancer, DErrico Gastric.

\section{UALCAN}

UALCAN (http://ualcan.path.uab.edu) is an open access database for analyzing cancer OMICS data from the The Cancer Genome Atlas (TCGA) [18]. Target genes can be tested for biomarkers identification in various cancers. It also provides platform to evaluate gene expression in molecular subtypes of different cancers and corresponding adjacent normal tissues. We obtained expression data pertaining to gastric cancer, and we limited our search to stomach adenocarcinoma, Difference of transcriptional expression was compared by students' $\mathrm{t}$ test and $p<0.05$ was considered as statically significant.

\section{cBioPortal}

The cBioPortal for Cancer Genomics (http://cbioportal. org) is a comprehensive database provides resource for investigating and analyzing multidimensional cancer genomics data from clinical and basic researches [19]. We obtained expression data pertaining to gastric cancer, and we limited our search to stomach adenocarcinoma. In this study, we evaluated aberrant expression 
of DGAT1 in patients with gastric cancer based on TCGA database, difference of transcriptional expression was compared by students' $\mathrm{t}$ test and when a $p$ value < 0.05 , the difference was considered statically significant. Other detailed parameters could be found in the additional file.

\section{Survival analysis}

Kaplan-Meier plotter (http://kmplot.com/analysis/) is a useful online database for the evaluation of prognostic value of target genes in cancer patients [20]. We obtained expression data pertaining to gastric cancer, and we limited our search to stomach adenocarcinoma. Patient samples with poorly differentiated gastric cancer were divided into two groups according to the median expression of the target gene, and overall survival (OS), first progression (FP), and post progression survival (PPS) of patients were plotted to evaluate value of target genes between high expression group and low expression group, with the hazard ratio (HR) with 95\% confidence intervals (CIs) and log rank $p$ value.

\section{Systematic analysis of immune infiltration}

TIMER (https://cistrome.shinyapps.io/timer/) is a comprehensive resource for systematic analysis of immune infiltrating cells across diverse cancer types. TIMER web server allows users to input target genes to conveniently access the tumor immunological, clinical, and genomic features [21]. In this study, we obtained expression data pertaining to gastric cancer, and we limited our search to stomach adenocarcinoma. And we evaluated correlation between DGAT1 expression and tumor-infiltrating immune cells via "gene" modules. And "survival" module was used to explore the clinical relevance of tumor immune subsets. "SCNA" module provides the comparison of tumor infiltration levels among tumors with different somatic copy number alterations for target genes. The gene expression level was displayed with log2 RSEM. The hazard ratio (HR) and 95\% CI of the clinical relevance of one or more tumor immune subsets was assessed using the Cox's proportional hazards model, with split percentage of patients was set as $50 \%$, and survival time was set as 120 months.

\section{Sample collection}

The study protocols and consent forms were approved by The Ethics Review Board of First Hospital of Jilin University (number 2019-070). All participants were given written informed consent. Cancer tissue and corresponding adjacent mucosa were collected from 5 gastric cancer patients.

\section{Cell culture and drug treatment}

Human gastric cell lines (MKN45, cat. ZQ0457; AGS, cat. Zq0240) and human gastric mucosal epithelial cell line (GES-1, cat. ZQ0905) acquired from Shanghai Zhong Qiao Xin Zhou Biotechnology Co.,Ltd. (http:// www.zqxzbio.com/Index/index.html) in 2020. All cell lines were tested by PCR and found to be mycoplasma negative. All cell lines were authenticated by profiling of STRs (Short Tandem Repeats) analysis.

Gastric cancer cell line AGS and MKN45 were cultured at $37^{\circ} \mathrm{C}$ in a $5 \% \mathrm{CO}_{2}$ atmosphere in RPMI 1640 medium supplemented with $10 \%$ fetal bovine serum (Gibco, USA) and penicillin/streptomycin $(100 \mathrm{U} / \mathrm{ml} / 100 \mu \mathrm{g} / \mathrm{ml}$, Yeasen, ShangHai, CN). Human gastric mucosal epithelial cell GES-1 was cultured at $37^{\circ} \mathrm{C}$ in a $5 \% \mathrm{CO}_{2}$ atmosphere in DMEM medium supplemented with $10 \%$ fetal bovine serum (Gibco, USA) and penicillin/streptomycin (100 U/ $\mathrm{ml} / 100 \mu \mathrm{g} / \mathrm{ml}$, Yeasen, ShangHai, CN).

Sodium oleate was purchased from Shanghai Aladdin (https://www.aladdin-e.com/), dissolved with heated double distilled water upon received, and then filtered with $0.22 \mu \mathrm{M}$ filter. Stock solution $(200 \mathrm{mM})$ stored aliquots at $-80{ }^{\circ} \mathrm{C}$ under sterile conditions. DGAT1 inhibitor A922500 was purchased from the selleck, China (https://www.selleck.cn/), dissolved with dimethylsulfoxide upon received. Stock solution $(100 \mathrm{mM})$ stored aliquots at $-80^{\circ} \mathrm{C}$ under sterile conditions.

$5 \times 10^{5}$ indicated cells were cultured in 6 well plates and treated with sodium oleate $(200 \mu \mathrm{M})$ and/or A922500 $(100 \mu \mathrm{M})$ for $12 \mathrm{~h}$

\section{RNA extraction and quantitative real-time PCR}

Total RNA was extracted from cells stimulated with sodium oleate and/or A922500 for $12 \mathrm{~h}$ with Trizol (Invitrogen) regent. Then cDNA was synthesized using TransScript First-Strand cDNA Synthesis SuperMix (TransGen Biotech). qRT-PCR was performed with an ABI StepOnePlus system (Applied Biosystems) with a SYBR Green Kit (TransGen Biotech). The expression level was normalized against the $\beta$-actin. Relative mRNA expression was calculated using the $2-\Delta \Delta C T$ method. The primer sequence sets used for DGAT1, NOX2, IDO, and $\beta$-actin was listed as follows:

\begin{tabular}{ll}
\hline Primer name & Primer sequence \\
\hline h/m-actin & F: 5' TTCAACACCCCAGCCATG 3' \\
& R: 5' CCTCGTAGATGGGCACAGT 3' \\
h-DGAT1 & F: 5' TATTGCGGCCAATGTCTTGC 3 \\
& R: 5'CACTGGAGTGATAGACTCAACCA 3' \\
h-NOX2 & F: 5' CACAGGCCTGAAACAAAAGA 3' \\
& R: 5' GCTTCAGGTCCACAGAGGAA 3' \\
h-IDO & F:5' GCCCTTCAAGTGTTCACCAA 3' \\
& R:5' GCCTTCCAGCCAGACAAATAT 3' \\
\hline
\end{tabular}

\section{Cell apoptosis analysis}

The MKN45 cells were treated with sodium oleate and/or A922500 for $24 \mathrm{~h}$. Then cells were collected 
and suspended in $100 \mu \mathrm{l}$ incubation buffer, stained with Annexin V-fluorescein isothiocyanate (FITC, $5 \mu \mathrm{l}$ ) and propidium iodide (PI, $10 \mu \mathrm{l})$ for $15 \mathrm{~min}$ at room temperature, then $400 \mu \mathrm{l}$ Binding Buffer was added to cells and cell apoptosis was then analyzed using an Ariall flow cytometer (BD Biosciences). Data were evaluated using FlowJo software (Version 10; FlowJo).

\section{Statistical analysis}

Significance was determined with the independentsamples Student's t-test analysis. Where indicated, statistical analysis was performed on Prism 7.0 (GraphPad Software), and $p<0.05$ was considered statistically significant. Data are representative of three independent experiments with similar results. Quantification of signal was shown in bar graphs and error bars represent mean $\pm \mathrm{SD}$.

\section{Results}

Abnormally elevated expression of DGAT1 in patients with gastric cancer

We initially investigated expression of DGAT1 in pancancer of patients by collecting data from the Oncomine database. DGAT1 level elevated in multiple cancer, such as bladder cancer, myeloma, pancreatic adenocarcinoma, papillary renal cell carcinoma, ovarian cancer, and gastric cancer (Fig. 1a). Among which, DGAT1 was highly expressed in patients with gastric cancer. Interestingly, the expression of DGAT1 had no significant difference between normal tissues and primary tumors in stomach adenocarcinoma patients (Fig. 1b). Next, we analyzed $D G A T 1$ level in cancer tissues upon tumor grades or individual cancer stages, and the results showed that during the deterioration of diseases, DGAT1 decreased in transcript level (Fig. 1c, d). Collectively, DGAT1 was highly expressed in gastric cancer tissues, and significantly decreased with the deterioration of diseases.

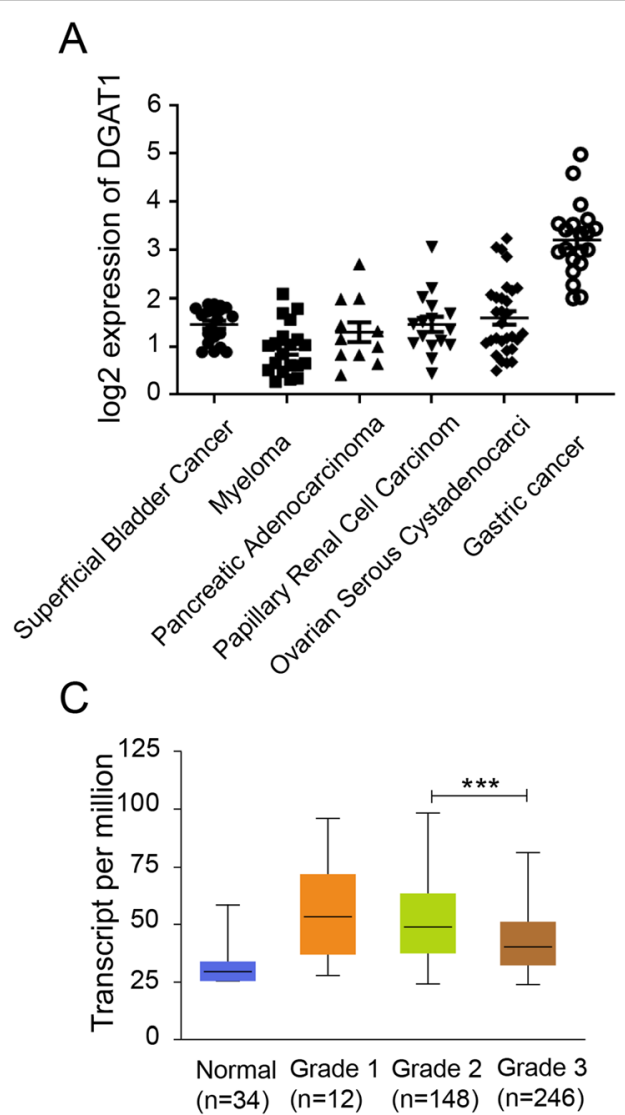

B
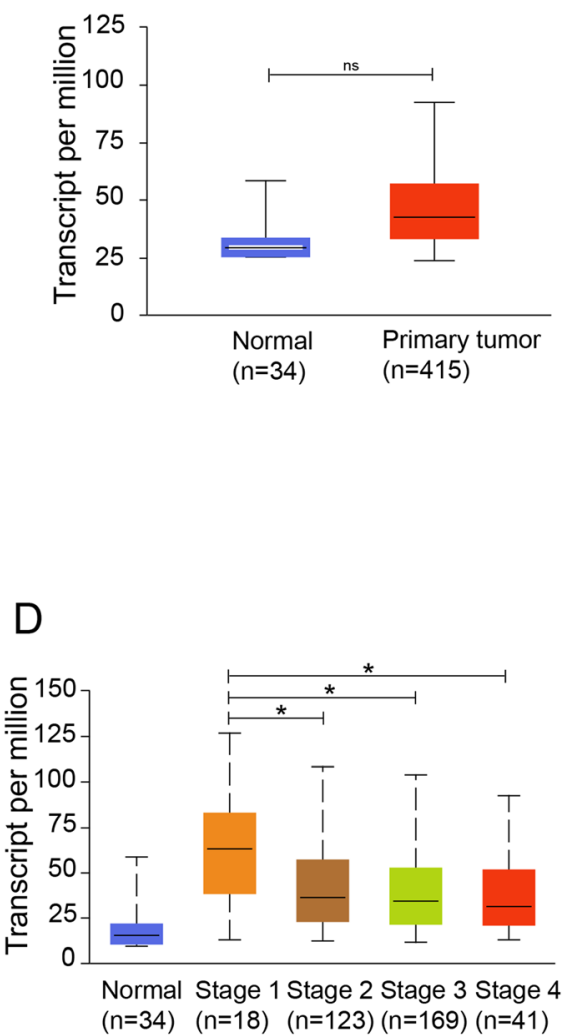

Fig. 1 The expression of DGAT1 was elevated in gastric cancer patients and then decreased with cancer progression. a The expression of DGAT1 in pan-cancer (Superficial Bladder Cancer, $n=20$; Myeloma, $n=20$; Pancreatic Adenocarcinoma, $n=11$; Papillary Renal Cell Carcinom, $n=16$; Ovarian Serous Cystadenocarcinoma, $n=29$; Gastric cancer, $n=20)$. b Comparison of DGAT1 expression between normal tissues and primary tumors in stomach adenocarcinoma patients ( $p=0.25054)$. Comparison of DGAT1 expression upon different tumor grades (c) or individual cancer stages (d). ${ }^{*} p<0.05 ;{ }^{* * *} p<0.001$. Tumor grades details: Grade 1: well differentiated (low grade); Grade 2: moderately differentiated (intermediate grade); Grade 3: poorly differentiated (high grade); Grade 4: undifferentiated (high grade). Data in (a) were collected from the Oncomine database, Data in (b-d) were collected from the UALCAN database 
Up-regulation of DGAT1 associated with poor prognosis in patients with gastric cancer

$D G A T 1$ is a crucial gene involved in the conversion of diacylglycerol and fatty acyl-CoA to triacylglycerol. Deletion and aberrant amplification of DGAT1 can be found in different types of the gastric cancer patients (Fig. 2a). To investigate the influence of altered expression of $D G A T 1$ to patients with gastric cancer, we compared survival time of patients depended on DGAT1 expression level. We performed survival analysis using Kaplanmeier plots and the results showed that higher expression of DGAT1 leads to lower overall survival (OS) rate in patients with poorly differentiated gastric cancer (Fig. 2b). Unexpectedly, survival curve analysis based on first progression analysis showed that elevated DGAT1 expression in cancer tissues has no impact on the survival time of the indicated patients (Fig. 2c). In addition, analysis based on post progression survival showed that survival curve of two groups based on DGAT1 expression had no significant difference (Fig. 2d). Collectively, increased DGAT1 expression can be detected in all types of gastric cancer patients, and high level of DGAT1 indicated a poor prognosis in gastric cancer patients.

\section{GO and KEGG pathway enrichment analyses of DGAT1}

$D G A T 1$ is a metabolism related enzyme involved in multiple biological processes. To systematically understand role of DGAT1 in cancer progression, we

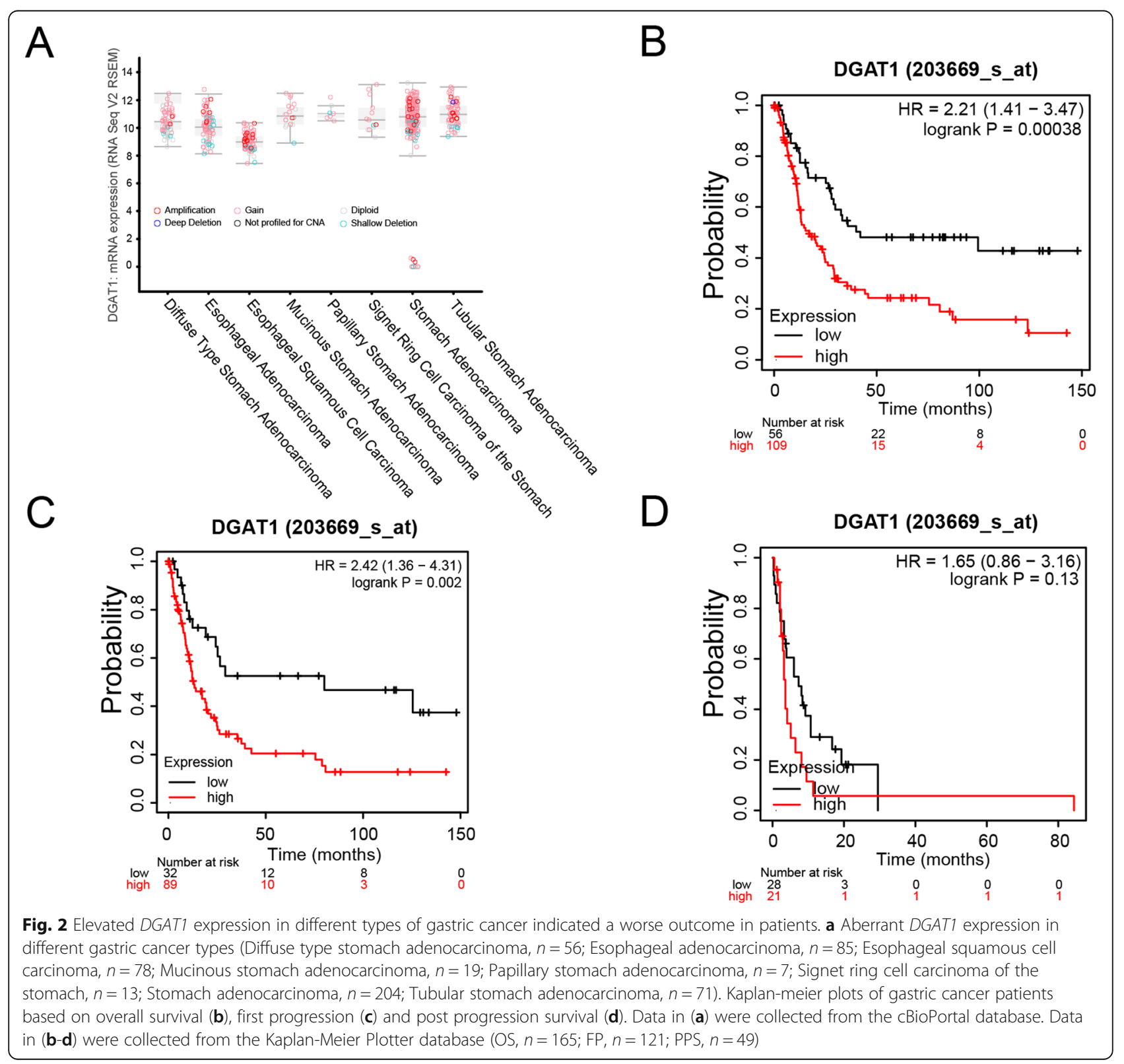


performed GO functional enrichments and KEGG pathway analysis by using the KOBAS online analysis database (http://kobas.cbi.pku.edu.cn/kobas3), with a $p$ value of $<0.05$ were obtained. The results were shown in Table 1 and Table 2 (Supplementary file), DGAT1 was mainly enriched in o-acyltransferase activity, transferase activity, transferring acyl groups, and triglyceride biosynthetic process. While the most significantly enriched pathways of the DGAT1 were Acyl chain remodeling of DAG and TAG, triglyceride biosynthesis and metabolism, and Fat digestion and absorption. These results indicated that DGAT1 play a key crucial role in lipid metabolism.

\section{DGAT1-expressing tumor-associated macrophage were} associated with poor OS in patients with gastric cancer DGAT1 can be expressed by multiple types of cells and participate in the regulation of energy metabolism. We analyzed DGAT1 levels in tumor-infiltrating immune cells in patients with gastric cancer. And the results revealed that mRNA expression and DNA copy number variation of DGAT1 was increased in immune cells of gastric cancer tissue other than immune cells in normal tissues (Fig. 3a, b). Next, we explored the clinical relevance of DGAT1 and several key tumor-infiltrating immune cell subsets under a multivariable Cox proportional hazard model. Interestingly, abnormal elevated DGAT1 in myeloid cells, especially macrophage, was significantly associated with reduced overall survival in patients with gastric cancer (Fig. 3c), indicated that DGAT1 could modulate the property of some immune cells and influence the prognosis of patients in an indirect way.

\section{DGAT1 increased level of reactive oxygen related genes in MKN45 cells}

As high level of DGAT1 in gastric cancer patients indicates a poor outcome with respect to overall survival, we firstly analyzed DGAT1 expression level in cancer tissue from gastric cancer patients. Results showed that DGAT1 level was increased in the cancer tissues (Fig. 4a). Next, we investigated DGAT1 level in several gastric cancer cell lines, and human gastric mucosal epithelial cell line GES-1 was used as normal control. As shown in Fig. 4b, DGAT1 expression was significantly higher in gastric cancer cell lines AGS and MKN45. Next, we wonder if block DGAT1 could inhibit tumor cell growth and metabolism. As shown in Fig. 4c, oleate sodium treatment increased DGAT1 expression in gastric cancer cell line MKN45. Surprisingly, A922500 treatment also inhibited expression of NOX2 and indoleamine 2,3-dioxygenase (IDO) in MKN45 (Fig. 4d, e), two genes correlated with proliferation and migration of gastric tumor cells $[15,16]$. Collectively, oleate sodium elevated DGAT1 expression in gastric cancer cell line MKN45 and DGAT1 blockade induced cell apoptosis in vitro.

\section{DGAT1 inhibition induced gastric cancer cell line MKN45 apoptosis}

As block DGAT1 effectively suppressed functional factors secretion in gastric cancer cell line, next we investigated cell viability after DGAT1 inhibition. The results showed that addition of A922500 treatment led to increased early apoptosis and necrosis in MKN45 cells (Fig. 5a, b). To validate the effect of iDGAT on MKN45 cells, sodium oleate was added to elevate DGAT1 level in target cells, then A922500 was added to block DGAT1 in MKN45 cells. Remarkably, A922500 treatment in the presence of sodium oleate induced cell apoptosis and necrosis (Fig. 5a, b), which again proved that high level of DGAT1 might facilitate the tumor growth and inhibition of increased DGAT1 expression effectively suppressed cell expansion in vitro.

\section{Discussion}

One of the principals aims of our study was to investigate the predicted biomarker of gastric cancer in human, and the potential target that facilitates to diagnosis and treatment gastric cancer. In this study, collected data from TCGA database reported abnormally elevated expression of DGAT1 in several types of cancer in patients, and we observed dramatic elevated level of DGAT1 in gastric cancer patients. Furthermore, KaplanMeier curves showed that aberrant expression of DGAT1 in immune cells and tumor tissues predict a poor prognosis in gastric cancer patients, and administration of iDGAT1 effectively inhibited reactive oxygen species expression in gastric cancer cell line, suggesting the considerable interest in DGAT1 as a potential target for the diagnosis and treatment of gastric cancer, which indicated a potential clinical impact for controlling gastric cancer.

Previous studies have shown that DGAT1 belongs to the membrane-bound O-acyltransferase superfamily, an acyltransferase involved in triacylglycerides synthesis and insufficient energy intake lead to triacylglycerides lipolysis [22]. During starvation, lipid droplets reduced lipotoxicity induced autophagic degradation of membranous organelles in DGAT1 depended manner [23]. In addition, mouse with DGAT1 deficiency are still viable but show alleviated ability to store triacylglycerols [24]. Important role of DGAT1 in energy metabolism indicated an active role of which in rapidly proliferating cells, such as tumor cells. Indeed, we observed highly expression of DGAT1 in several types of cancer in patients, which was especially correlated with worse prognosis in gastric cancer patients. Surprisingly, we found 
A
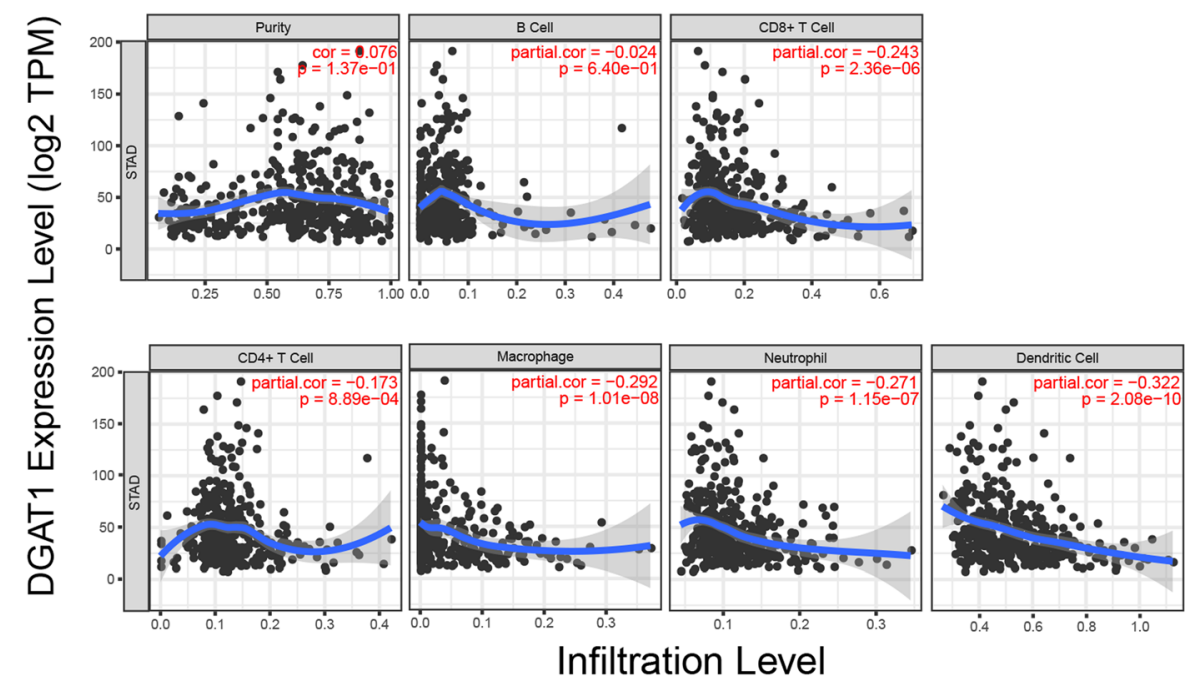

B

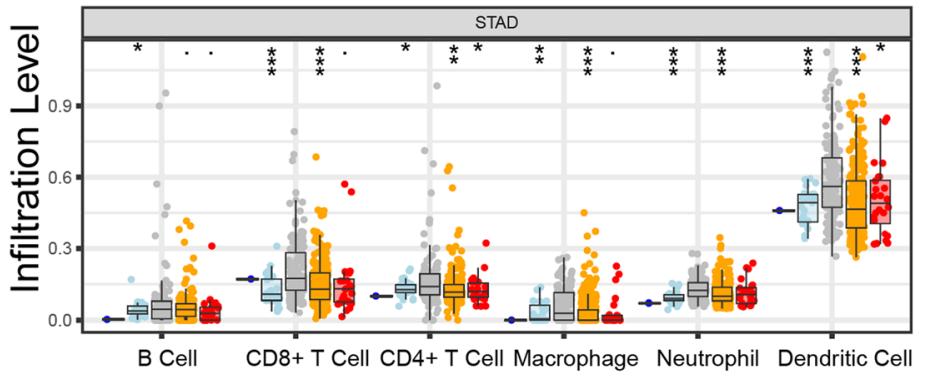

Copy Number

Deep Deletion

Arm-level Gain

- High Amplication

C

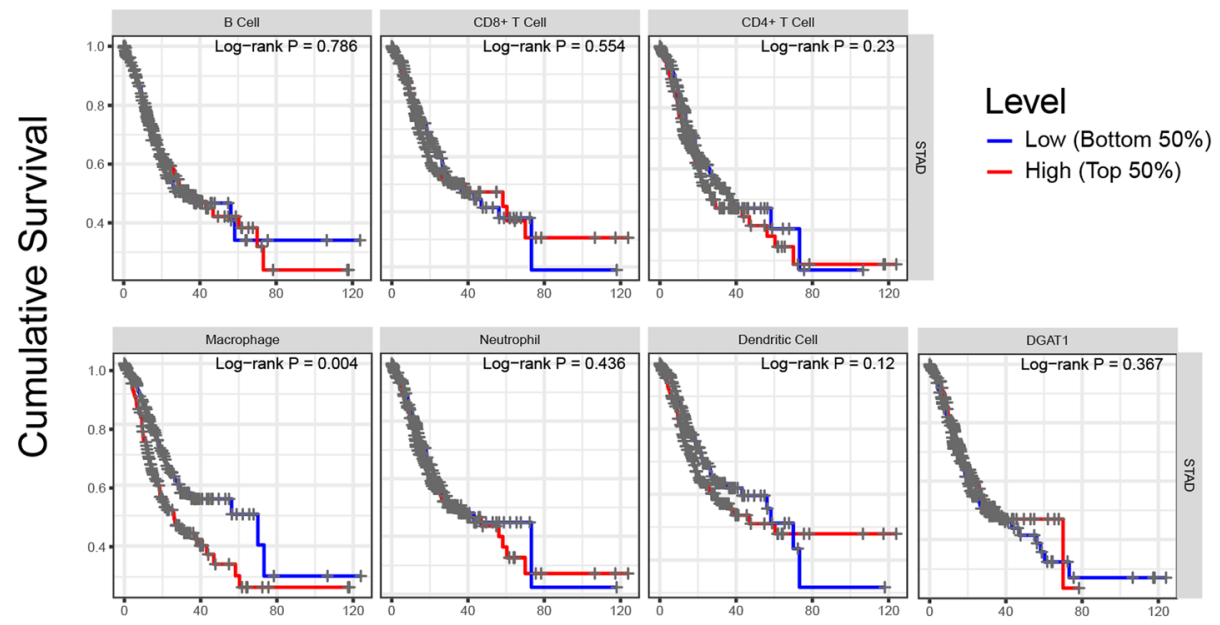

Time to Follow-Up (months)

Fig. 3 Aberrantly elevated expression of DGAT1 in tumor-infiltrating macrophages were correlated with poor prognosis in gastric cancer patients. a DGAT1 expression was significantly negatively correlated with the levels of infiltrating $C D 8^{+} T$ cells, $C D 4^{+} T$ cells, macrophages, neutrophils, and dendritic cells in STAD. b Comparison of tumor infiltration levels in gastric cancer with different somatic copy number alterations for DGAT1. c Increased DGAT1 expression in tumor-infiltrating macrophages were correlated with reduced overall survival in gastric cancer patients. Data in (ac) were collected from the TIMER database 
A

$\mathrm{D}$
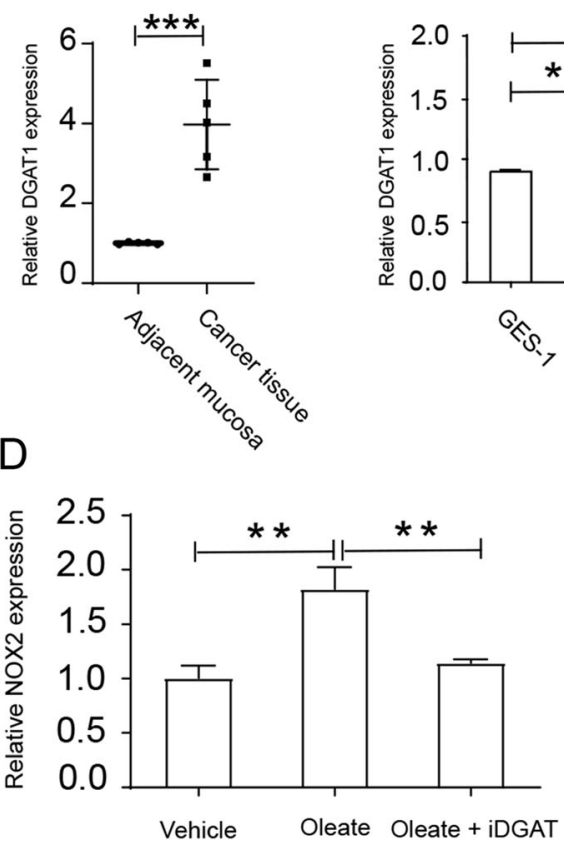

B

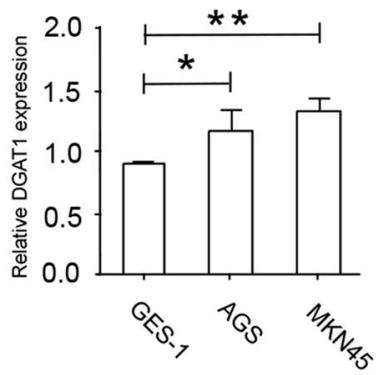

C

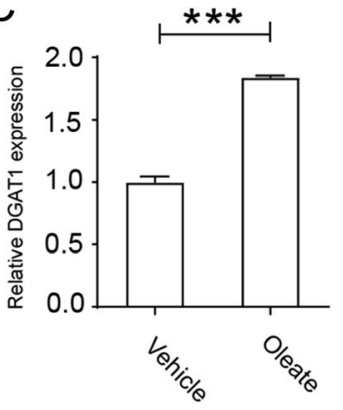

$\mathrm{E}$

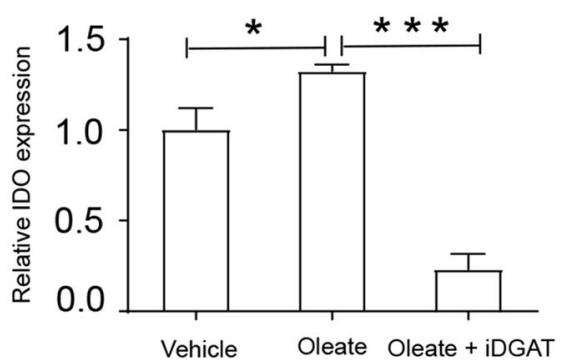

Fig. 4 Blocking DGAT1 pathway impaired reactive oxygen species expression in gastric cancer cell lines. a Gastric cancer tissues expressed higher level of DGAT1 $(n=5, p=0.0004) .5 \times 10^{5}$ GES-1, AGS or MKN45 cells were cultured in 6 well plates and/or treated with sodium oleate $(200 \mu M)$ and/or A922500 (100 $\mu \mathrm{M})$ for $12 \mathrm{~h}$. Then cells were collected and mRNAs were isolated for further studies. qPCR analysis of DGAT1 (b) in gastric cancer cell lines, or in MKN45 after sodium oleate treatment (c), or NOX2 (d) and IDO (e) in MKN45 after sodium oleate and/or iDGAT treatment. ${ }^{*} P<0.05 ;{ }^{*} P<0.01 ;{ }^{* *} P<0.001$. Data are representative of three independent experiments with similar results. Quantification of signal was shown in bar graphs and error bars represent mean \pm SD

A

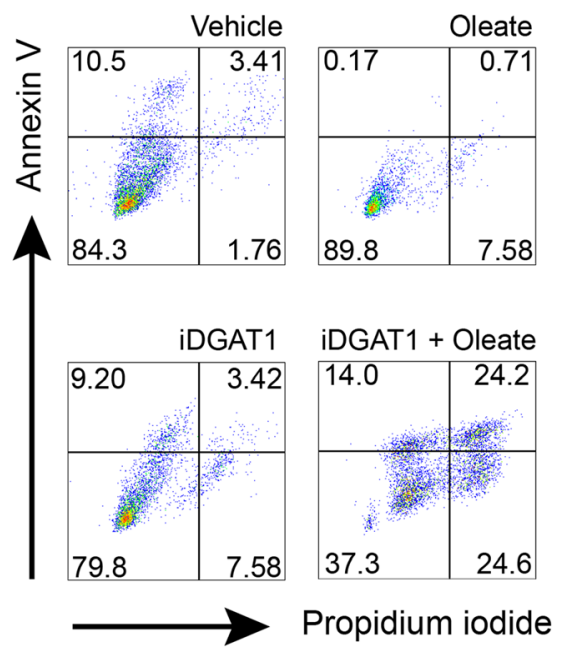

B

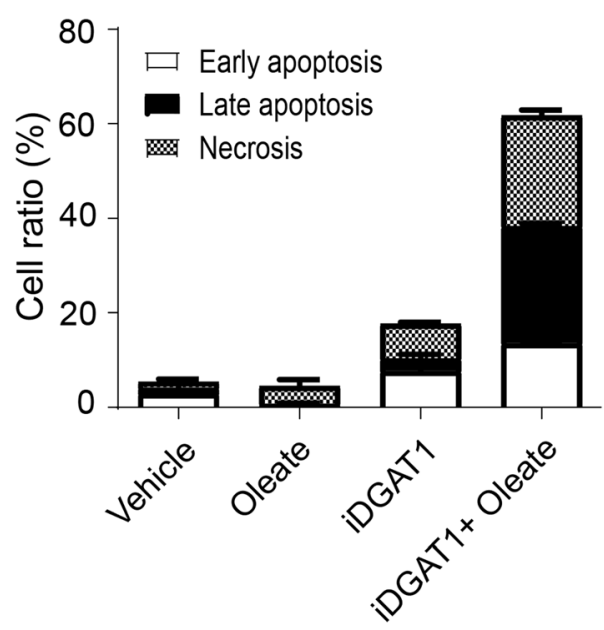

Fig. 5 Blocking DGAT1 lead to MKN45 cells apoptosis in vitro. $5 \times 10^{5}$ MKN45 cells were cultured in 6 well plates and treated with sodium oleate $(200 \mu \mathrm{M})$ and/or A922500 (100 $\mathrm{\mu M})$ for $24 \mathrm{~h}$. Then cells were collected and stained with indicated reagents for apoptosis detection. Cell apoptosis of MKN45 cells with sodium oleate and/or A922500 stimulation as examined by annexin V/PI staining (a, b). Data are representative of three independent experiments with similar results. Quantification of signal was shown in bar graphs and error bars represent mean \pm SD 
the elevated expression of DGAT1 in tumor infiltrating macrophage also showed negative correlation with poor overall survival in stomach adenocarcinoma patients. Although significantly fewer studies have functionally evaluated DGAT1 in cancer progression and metabolism, the available data reported that DGAT1 indirectly promoted tumor growth via modulating lipid droplets formation in macrophages and enhancing suppressive function of myeloid cells to inhibit immune response in vitro [10]. Another group demonstrated that inhibition of DGAT1 reduced prostate tumor growth [13], indicated that DGAT1 could support tumor growth both directly and indirectly.

Myeloid cells produce large amount of reactive oxygen species to regulate inflammatory response. The phagocyte NADPH oxidase (NOX2) is mainly expressed by macrophages, neutrophils, and DCs [25], play a key role in antimicrobial immune response after activating pathogen recognition receptors expressed by those phagocytes [26]. Prior studies have shown that monocytic acute myeloid leukemia cells promotes itself survival in a NOX2 depended manner, blocking NOX2 efficiently inhibits acute myeloid leukemia cells proliferation [27, 28]. An in vitro study showed that NOX2-ROS activates multiple pathways to promotes gastric cancer cells proliferation and migration [16]. In current study, we detected sodium oleate treatment improved DGAT1 expression in gastric cancer cell line MKN45, and NOX2 and IDO expression level also increased significantly. Meanwhile blocked DGAT1 by the A922500 dramatically inhibited NOX2 and IDO level, and cells apoptosis detection showed an increased apoptotic ratio when compared that with control groups. Similar with NOX2, prior studies that have noted the importance of IDO in promoting tolerogenic responses under tumor inflammatory condition. In addition, IDO could be expressed by several immune regulatory cells, such as tumor-infiltrating myeloid-derived suppressor cells, that is crucial in the establishment and maintenance of cancer immune tolerance in tumor microenvironment [29]. Our study confirms that IDO is associated with tumor cells survival and inhibits IDO expression led to tumor cell apoptosis [30]. Thus, the present study raises the possibility that DGAT1 could be a promising biomarker and potential target for the diagnosis and treatment of gastric cancer.

\section{Conclusions}

The current study describes the crucial role of DGAT1 in gastric cancer progression. We demonstrated a worse prognosis in gastric cancer patients with elevated DGAT1 expression. Intervention strategies blocking $D G A T 1$ in patients might be clinically valuable in the future gastric cancer treatment.

\section{Abbreviations}

DGAT1: Diacylglycerol-acyltransferase 1; OS: Overall survival; NOX2: NADPH oxidase 2; IDO: Indoleamine 2,3-dioxygenase; GO: Gene ontology;

KEGG: Kyoto Encyclopedia of Genes and Genomes

\section{Supplementary Information}

The online version contains supplementary material available at https://doi. org/10.1186/s12885-021-07976-5.

\section{Additional file 1.}

Acknowledgements

Not applicable.

\section{Authors' contributions}

YX and ZM conceived and designed the experiments. PH, SC, and FH performed the experiments and analyzed the sequencing data. PH wrote the manuscript. YX carried out the revising process. $\mathrm{PH}, \mathrm{FH}$, and ZM participated in the sample collecting, data analysis, and interpretation of data. All authors read and approved the final manuscript.

\section{Funding}

This work was supported by the grants from the Science and Technology Department of Jilin Province (20190201140JC to H.Y.) The funders had no role in study design, data collection and analysis, decision to publish, or preparation of the manuscript.

\section{Availability of data and materials}

The datasets used and/or analyzed during the current study are available from the corresponding authors on reasonable request.

\section{Declarations}

Ethics approval and consent to participate

The current study has been submitted to and approved by the ethics committee of the First Hospital of Jilin University. All participants were given written informed consent. The cell lines used in this study has been approved by the ethics committee of the First Hospital of Jilin University.

\section{Consent for publication}

Not applicable.

\section{Competing interests}

The authors declare no competing interests.

\section{Author details}

${ }^{1}$ Department of Gastroenterology, The First Hospital of Jilin University, Changchun 130021, Jilin, China. ${ }^{2}$ Department of Rehabilitation, The First Hospital of Jilin University, Changchun, Jilin 130021, People's Republic of China. ${ }^{3}$ Department of Hepatology and Gastroenterology, The Second Part of First Hospital of Jilin University, Changchun, China. ${ }^{4}$ Central Laboratory, The First Hospital of Jilin University, Changchun, Jilin, China. ${ }^{5}$ Key Laboratory of Organ Regeneration and Transplantation, Ministry of Education, Changchun 130021, Jilin, China.

Received: 24 August 2020 Accepted: 25 February 2021

Published online: 09 March 2021

\section{References}

1. Bray F, Ferlay J, Soerjomataram I, Siegel RL, Torre LA, Jemal A. Global cancer statistics 2018: GLOBOCAN estimates of incidence and mortality worldwide for 36 cancers in 185 countries. CA Cancer J Clin. 2018;68(6):394-424.

2. Karimi P, Islami F, Anandasabapathy S, Freedman ND, Kamangar F. Gastric cancer: descriptive epidemiology, risk factors, screening, and prevention. Cancer Epidemiol Biomarkers Prev. 2014;23(5):700-13.

3. Goetze OT, Al-Batran SE, Chevallay M, Mönig SP. Multimodal treatment in locally advanced gastric cancer. Updat Surg. 2018;70(2):173-9.

4. Herszényi L, Tulassay Z. Epidemiology of gastrointestinal and liver tumors. Eur Rev Med Pharmacol Sci. 2010;14(4):249-58. 
5. Correa P. Gastric cancer: overview. Gastroenterol Clin N Am. 2013;42(2): 211-7.

6. Pasechnikov V, Chukov S, Fedorov E, Kikuste I, Leja M. Gastric cancer: prevention, screening and early diagnosis. World J Gastroenterol. 2014; 20(38):13842-62.

7. Heeren J, Scheja L. Brown adipose tissue and lipid metabolism. Curr Opin Lipidol. 2018;29(3):180-5.

8. Singh AK, Aryal B, Chaube B, Rotllan N, Varela L, Horvath TL, Suárez Y, Fernández-Hernando C. Brown adipose tissue derived ANGPTL4 controls glucose and lipid metabolism and regulates thermogenesis. Mol Metab. 2018;11:59-69.

9. Padanad MS, Konstantinidou G, Venkateswaran N, Melegari M, Rindhe S, Mitsche M, Yang C, Batten K, Huffman KE, Liu J, et al. Fatty Acid Oxidation Mediated by Acyl-CoA Synthetase Long Chain 3 Is Required for Mutant KRAS Lung Tumorigenesis. Cell Rep. 2016;16(6):1614-28.

10. Wu H, Han Y, Rodriguez Sillke Y, Deng H, Siddiqui S, Treese C, Schmidt F, Friedrich $M$, Keye J, Wan J, et al. Lipid droplet-dependent fatty acid metabolism controls the immune suppressive phenotype of tumorassociated macrophages. EMBO Mol Med. 2019;11(11):e10698.

11. Bhatt-Wessel B, Jordan TW, Miller JH, Peng L. Role of DGAT enzymes in triacylglycerol metabolism. Arch Biochem Biophys. 2018;655:1-11.

12. Mitra R, Le T, Gorjala P, Goodman OB, Jr. Positive regulation of prostate cancer cell growth by lipid droplet forming and processing enzymes DGAT1 and ABHD5. BMC Cancer. 2017;17(1):631.

13. Nardi F, Franco OE, Fitchev P, Morales A, Vickman RE, Hayward SW, Crawford SE. DGAT1 Inhibitor Suppresses Prostate Tumor Growth and Migration by Regulating Intracellular Lipids and Non-Centrosomal MTOC Protein GM130. Sci Rep. 2019;9(1):3035.

14. Liu H, Shen Z, Wang Z, Wang X, Zhang H, Qin J, Qin X, Xu J, Sun Y. Increased expression of IDO associates with poor postoperative clinical outcome of patients with gastric adenocarcinoma. Sci Rep. 2016;6: 21319.

15. Kim SM, Hur DY, Hong SW, Kim JH. EBV-encoded EBNA1 regulates cell viability by modulating miR34a-NOX2-ROS signaling in gastric cancer cells. Biochem Biophys Res Commun. 2017:494(3-4):550-5.

16. Wang Z, Tang T, Wang S, Cai T, Tao H, Zhang Q, Qi S, Qi Z. Aloin inhibits the proliferation and migration of gastric Cancer cells by regulating NOX2ROS-mediated pro-survival signal pathways. Drug Des Devel Ther. 2020;14: $145-55$.

17. Rhodes DR, Yu J, Shanker K, Deshpande N, Varambally R, Ghosh D, Barrette T, Pandey A, Chinnaiyan AM. ONCOMINE: a cancer microarray database and integrated data-mining platform. Neoplasia. 2004;6(1):1-6.

18. Chandrashekar DS, Bashel B, Balasubramanya SAH, Creighton CJ, PonceRodriguez I, Chakravarthi B, Varambally S. UALCAN: a portal for facilitating tumor subgroup gene expression and survival analyses. Neoplasia. 2017; 19(8):649-58.

19. Cerami E, Gao J, Dogrusoz U, Gross BE, Sumer SO, Aksoy BA, Jacobsen A, Byrne CJ, Heuer ML, Larsson E, et al. The cBio cancer genomics portal: an open platform for exploring multidimensional cancer genomics data. Cancer Discov. 2012;2(5):401-4.

20. Nagy Á, Lánczky A, Menyhárt O, Győrffy B. Validation of miRNA prognostic power in hepatocellular carcinoma using expression data of independent datasets. Sci Rep. 2018;8(1):9227.

21. Li T, Fan J, Wang B, Traugh N, Chen Q, Liu JS, Li B, Liu XS. TIMER: a web server for comprehensive analysis of tumor-infiltrating immune cells. Cancer Res. 2017;77(21):e108-10.

22. Chitraju C, Mejhert N, Haas JT, Diaz-Ramirez LG, Grueter CA, Imbriglio JE, Pinto S, Koliwad SK, Walther TC, Farese RV Jr. Triglyceride synthesis by DGAT1 protects adipocytes from lipid-induced ER stress during lipolysis. Cell Metab. 2017;26(2):407-18 e403.

23. Nguyen TB, Louie SM, Daniele JR, Tran Q, Dillin A, Zoncu R, Nomura DK, Olzmann JA. DGAT1-Dependent Lipid Droplet Biogenesis Protects Mitochondrial Function during Starvation-Induced Autophagy. Dev Cell. 2017:42(1):9-21.e25.

24. Smith SJ, Cases S, Jensen DR, Chen HC, Sande E, Tow B, Sanan DA, Raber J, Eckel RH, Farese RV Jr. Obesity resistance and multiple mechanisms of triglyceride synthesis in mice lacking Dgat. Nat Genet. 2000;25(1):87-90.

25. Singel $\mathrm{KL}$, Segal BH. NOX2-dependent regulation of inflammation. Clin Sci. 2016;130(7):479-90.

26. Kumar A, Barrett JP, Alvarez-Croda DM, Stoica BA, Faden Al, Loane DJ. NOX2 drives M1-like microglial/macrophage activation and neurodegeneration following experimental traumatic brain injury. Brain Behav Immun. 2016;58: 291-309.

27. Martner A, Thorén FB, Aurelius J, Hellstrand K. Immunotherapeutic strategies for relapse control in acute myeloid leukemia. Blood Rev. 2013:27(5):209-16.

28. Kiffin $\mathrm{R}$, Grauers Wiktorin H, Nilsson MS, Aurelius J, Aydin E, Lenox B, Nilsson JA, Ståhlberg A, Thorén FB, Hellstrand K, et al. Anti-Leukemic Properties of Histamine in Monocytic Leukemia: The Role of NOX2. Front Oncol. 2018;8: 218.

29. Li F, Zhao Y, Wei L, Li S, Liu J. Tumor-infiltrating Treg, MDSC, and IDO expression associated with outcomes of neoadjuvant chemotherapy of breast cancer. Cancer Biol Ther. 2018;19(8):695-705.

30. Seeber A, Klinglmair G, Fritz J, Steinkohl F, Zimmer KC, Aigner F, Horninger W, Gastl G, Zelger B, Brunner A, et al. High IDO-1 expression in tumor endothelial cells is associated with response to immunotherapy in metastatic renal cell carcinoma. Cancer Sci. 2018;109(5):1583-91.

\section{Publisher's Note}

Springer Nature remains neutral with regard to jurisdictional claims in published maps and institutional affiliations.

\section{Ready to submit your research? Choose BMC and benefit from:}

- fast, convenient online submission

- thorough peer review by experienced researchers in your field

- rapid publication on acceptance

- support for research data, including large and complex data types

- gold Open Access which fosters wider collaboration and increased citations

- maximum visibility for your research: over $100 \mathrm{M}$ website views per year

At BMC, research is always in progress.

Learn more biomedcentral.com/submissions 